In addition, the authors showed that the proliferative response of a human B-lineage leukemic cell line to SDF-1 is PKC- $\zeta$ dependent. It remains to be seen how this relates to the mechanism by which SDF-1 influences the cycling status of primitive normal cells, since it appears that the result can range from induced quiescence $(14,15)$ to enhanced turnover (16), depending on the type of progenitor being assessed, the context of its exposure, and/or the concentration of SDF-1 present.

\section{Clinical implications}

As pointed out by Petit et al. (13), pinpointing the molecular signaling mechanisms that mediate SDF-1 effects on primitive hematopoietic cells and leukemic cells may have important implications for future therapies. The present findings certainly introduce the possibility of considering new agents for improving stem cell mobilization regimes. More speculative is the concept of exploiting small molecule inhibitors of PKC- $\zeta$ to interfere with SDF-1promoted metastases. Since the initial report of a role of SDF-1 in breast cancer metastases (17), evidence that this pathway is hijacked in numerous other tumors has been obtained (18). The study by Petit et al. (13) has thus also set the stage for examining a new approach to the treatment of disseminating malignant cells.

Address correspondence to: Connie J. Eaves, Terry Fox Laboratory, 601 West 10th Avenue, Vancouver, British Columbia V5Z 1L3, Canada. Phone: (604) 877-6070; Fax: (604) 877-0712; E-mail: ceaves@bccrc.ca. regulates hematopoietic stem cell quiescence in the bone marrow niche. Cell. 118:139-140.

2. Wright, D.E., Wagers, A.J., Gulati, A.P., Johnson, F.L., and Weissman, I.L. 2001. Physiological migration of hematopoietic stem and progenitor cells. Science. 294:1933-1936.

3. Thomas, J., Liu, F., and Link, D.C. 2002. Mechanisms of mobilization of hematopoietic progenitors with granulocyte colony-stimulating factor. Curr. Opin. Hematol. 9:183-189.

4. Lapidot, T., and Kollet, O. 2002. The essential roles of the chemokine SDF-1 and its receptor CXCR4 in human stem cell homing and repopulation of transplanted immune-deficient NOD/SCID and NOD/ SCID/B2m ${ }^{\text {null }}$ mice. Leukemia. 16:1992-2003.

5. Tokoyoda, K., Egawa, T., Sugiyama, T., Choi, B.-I., and Nagasawa, T. 2004. Cellular niches controlling B lymphocyte behavior within bone marrow during development. Immunity. 20:707-718.

6. Papayannopoulou, T. 2004. Current mechanistic scenarios in hematopoietic stem/progenitor cell mobilization. Blood. 103:1580-1585.

7. Messner, H.A., Fauser, A.A., Lepine, J., and Martin, M. 1980. Properties of human pluripotent hemopoietic progenitors. Blood Cells. 6:595-607.

8. Eaves, C.J., and Eaves, A.C. 1987. Cell culture studies in CML [review]. Baillieres Clin. Haematol. 1:931-961.
9. Morrison, S.J., Wright, D.E., and Weissman, I.L. 1997. Cyclophosphamide/granulocyte colonystimulating factor induces hematopoietic stem cells to proliferate prior to mobilization. Proc. Natl. Acad. Sci. U. S. A. 94:1908-1913.

10. Iijima, M., Huang, Y.E., and Devreotes, P. 2002. Temporal and spatial regulation of chemotaxis. Dev. Cell. 3:469-478.

11. Toker, A. 2000. Protein kinases as mediators of phosphoinositide 3-kinase signaling. Mol. Pharmacol. 57:652-658.

12. Wang, J.F., Park, I.W., and Groopman, J.E. 2000. Stromal cell-derived factor- 1 alpha stimulates tyrosine phosphorylation of multiple focal adhesion proteins and induces migration of hematopoietic progenitor cells: roles of phosphoinositide- 3 kinase and protein kinase C. Blood. 95:2505-2513.

13. Petit, I., et al. 2005. Atypical PKC- $\zeta$ regulates SDF-1mediated migration and development of human CD34+ progenitor cells. J. Clin. Invest. 115:168-176. doi:10.1172/JCI200521773.

14. Cashman, J., Clark-Lewis, I., Eaves, A., and Eaves, C. 2002. Stromal-derived factor- 1 inhibits the cycling of very primitive human hematopoietic cells in vitro and in NOD/SCID mice. Blood. 99:792-799.

15. Cashman, J., Dykstra, B., Clark-Lewis, I., Eaves, A., and Eaves, C. 2002. Changes in the proliferative activity of human hematopoietic stem cells in NOD/SCID mice and enhancement of their transplantability after in vivo treatment with cell cycle inhibitors. J. Exp. Med. 196:1141-1149.

16. Lataillade, J.-J., et al. 2000. Chemokine SDF-1 enhances circulating $\mathrm{CD} 34^{+}$cell proliferation in synergy with cytokines: possible role in progenitor survival. Blood. 95:756-768.

17. Muller, A., et al. 2001. Involvement of chemokine receptors in breast cancer metastasis. Nature. 410:50-56.

18. Zlotnik, A. 2004. Chemokines in neoplastic progression. Sem. Cancer Biol. 14:181-185.

\title{
Mac the knife? Macrophages — the double-edged sword of hepatic fibrosis
}

\author{
Scott L. Friedman \\ Division of Liver Diseases, Mount Sinai School of Medicine, New York, New York, USA.
}

\begin{abstract}
Progression of hepatic fibrosis requires sustained inflammation leading to activation of stellate cells into a fibrogenic and proliferative cell type, whereas regression is associated with stellate cell apoptosis. The contribution of hepatic macrophages to these events has been largely overlooked. However, a study in this issue of the JCI demonstrates that macrophages play pivotal but divergent roles, favoring ECM accumulation during ongoing injury but enhancing matrix degradation during recovery (see the related article beginning on page 56). These findings underscore the potential importance of hepatic macrophages in regulating both stellate cell biology and ECM degradation during regression of hepatic fibrosis.
\end{abstract}

Nonstandard abbreviations used: $\mathrm{CCl}_{4}$, carbon tetrachloride; TIMP-1, tissue inhibitor of metalloproteinase-1; TRAIL, TNF-related apoptosis-inducing ligand.

Conflict of interest: The author has declared that no conflict of interest exists.

Citation for this article: J. Clin. Invest. 115:29-32 (2005). doi:10.1172/JCI200523928.
Kupffer cells, the resident macrophage population of the liver, have in recent years lost their leading position in the pecking order of cell types known to contribute to hepatic injury and repair. Long recognized for their activity in liver inflammation, they had been increasingly overlooked while a stellate cell- centric view of hepatic fibrosis had replaced the earlier focus on macrophages (Figure 1). Symbolic of this demotion, a biannual meeting initially convened as the International Kupffer Cell Symposium changed its name in 1990 to the International Symposium on Cells of the Hepatic Sinusoid (1). This evolution has been understandable because recent studies have established the activated hepatic stellate cell and its myofibroblast counterpart as the major sources of ECM in both experimental and human liver disease (2). As a result, a comprehensive picture of hepatic stellate cell activation in liver injury has emerged, resulting in exciting new prospects for targeting a range of growth factors, receptors, and intracellular mediators in the treatment of hepatic fibrosis (3). 


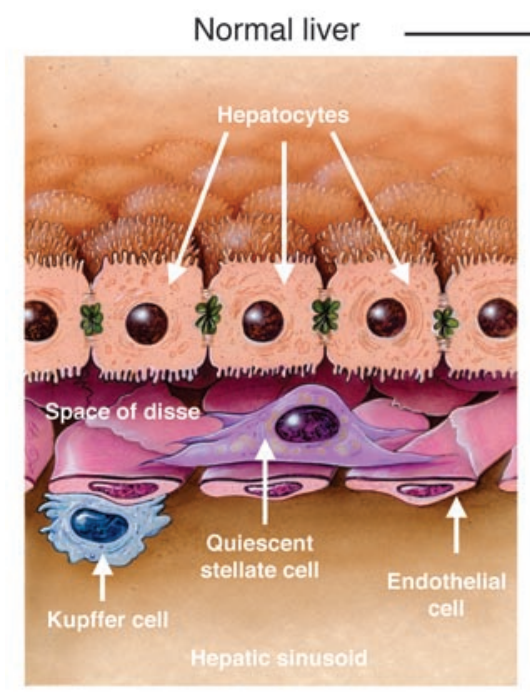

The paper by Duffield and colleagues in this issue of the JCI (4), however, firmly reestablishes the hepatic macrophage as a central determinant of the liver's response to injury and repair. The study is built upon 2 important concepts. First, macrophages have more than one pathway of activation and response, depending on the specific stimulus and biologic context; indeed, divergence of macrophage responses has been recognized for many years $(5,6)$ but has only recently been placed in a biologically coherent context that defines pro- and anti-inflammatory phenotypes. Proinflammatory actions of macrophages include antigen presentation,

\section{Figure 2}

The double-edged sword of hepatic macrophage activity in hepatic fibrosis progression versus recovery. The findings by Duffield et al. (4) in this issue of the $\mathrm{JCl}$ suggest that hepatic macrophages may induce divergent effects on liver fibrosis by promoting stellate cell activation in the face of continued injury and fibrosis; and stellate cell apoptosis during recovery associated with fibrosis regression as injury subsides. Evidence from other studies implicates TGF- $\beta 1$ as one potential paracrine stimulator of stellate cell activation by macrophages, while TRAIL may mediate stellate cell apoptosis during fibrosis regression associated with recovery. Apoptosis associated with the loss of TIMP-1 may unmask latent matrix protease activity released by either macrophages, stellate cells, or other cell types. It is not certain if the same macrophages account for the divergent activities of this cell type or whether different macrophage subsets mediate these opposing pathways.

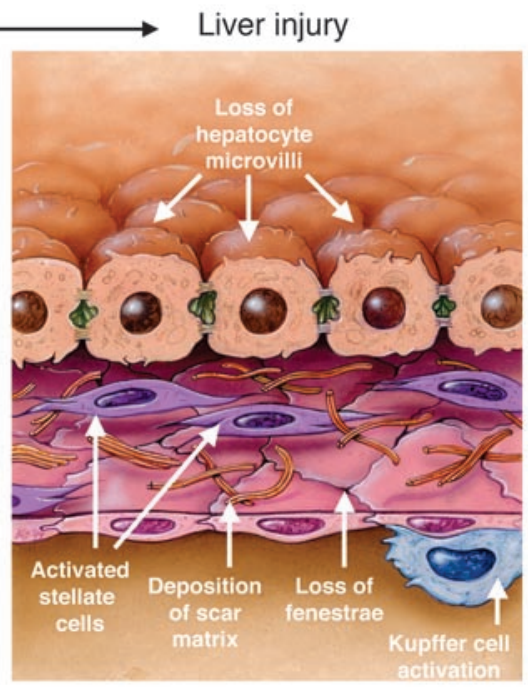

$\mathrm{T}$ cell activation, and cytokine and protease release, among others (7). However, antiinflammatory actions have been increasingly appreciated, particularly those induced by IL-4, including induction of immune tolerance (8), innate immunity (9), and T cell differentiation $(7,10)$.

A second, more recently developed concept addresses the behavior of hepatic stellate cells in the resolution of liver injury and fibrosis. Whereas initial interest in stellate cell biology focused on the cells' activation and fibrogenic properties during progressive liver injury, more recent efforts have explored both their fate as liver injury resolves and the

\section{Figure 1}

Cellular events in hepatic fibrosis. As fibrosis develops in response to liver injury, stellate cell activation leads to accumulation of scar matrix. This in turn contributes to the loss of hepatocyte microvilli and endothelial fenestrae, which results in the deterioration of hepatic function. Kupffer cell (macrophage) activation contributes to the activation of stellate cells. Figure modified with permission from The Journal of Biological Chemistry (24).

mechanisms underlying persistence of fibrosis in sustained liver injury. This is a vital area of inquiry since clarification of mechanisms by which the liver naturally restores its architecture might be exploited in developing antifibrotic therapies for patients with chronic liver disease. During regression of experimental liver fibrosis, activated stellate cells undergo programmed cell death associated with loss of tissue inhibitor of metalloproteinase-1 (TIMP-1) expression (11). Because TIMP-1 not only enhances stellate cell survival but also antagonizes matrix degradation, the loss of TIMP-1-expressing stellate cells is thought to unleash latent matrix-degrading activity, leading to the breakdown of scar matrix and the reconstitution of normal hepatic architecture. When liver injury persists, TIMP-1 levels remain high, and progressive cross-linking of collagen may render the accumulating matrix relatively insoluble to proteases $(12,13)$. This concept is reinforced by studies demonstrat-

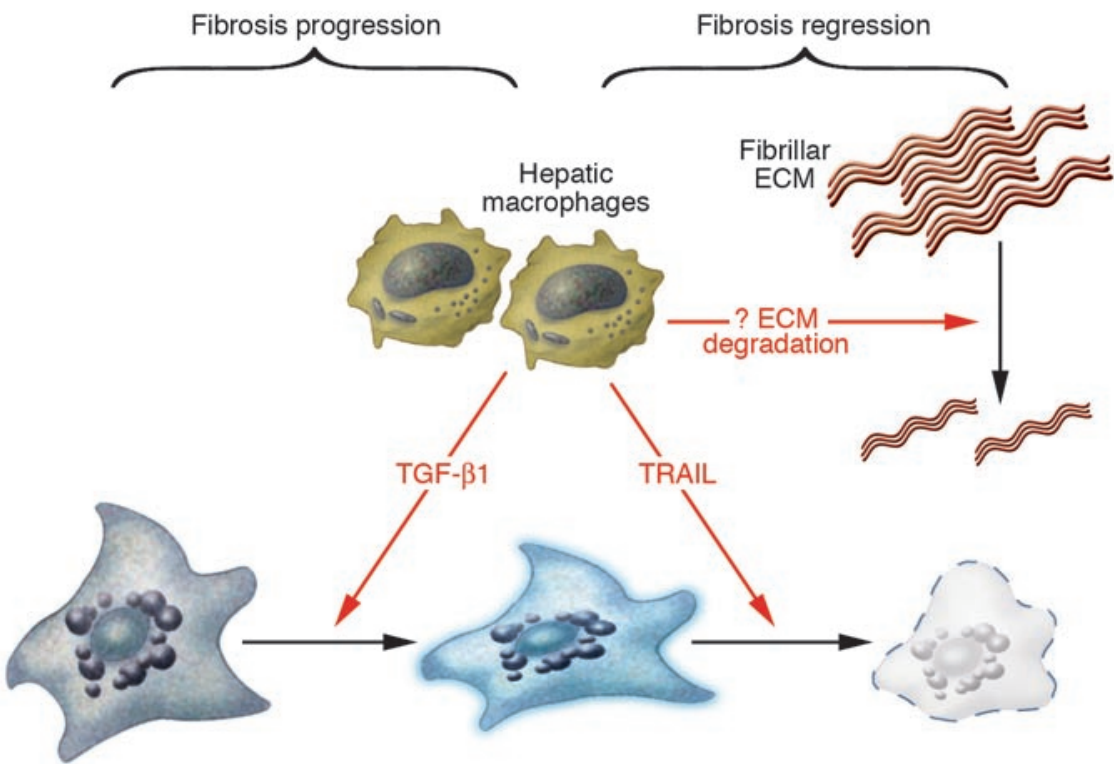

Quiescent stellate cell

Activated stellate cell / myofibroblast

Increased TIMP-1
Apoptotic stellate cell Decreased TIMP-1 
ing attenuation of fibrosis when TIMP-1 is inhibited (14). Significant gaps in this paradigm have persisted, however, including the source(s) and identity of salutary proteases in fibrosis resolution and the role of other cellular elements, including not only hepatic macrophages, but also sinusoidal endothelium.

\section{Macrophage depletion in mice identifies novel roles in regulating hepatic fibrosis}

Duffield and colleagues have generated a transgenic mouse model in which macrophages can be selectively depleted in a regulated manner (4). Expression of a human version of a diptheria toxin receptor driven by the promoter of a myeloid antigen (CD11b) renders transgenic macrophages (called CD11b-DTR cells) susceptible to killing by administration of diptheria toxin. Careful control experiments confirmed that susceptibility is macrophage specific and does not affect other cell lineages. Next the investigators compared the impact of macrophage depletion on hepatic fibrosis between a murine model in which macrophages were depleted during the progression of fibrosis by the administration of diptheria toxin after 12 weeks of carbon tetrachloride $\left(\mathrm{CCl}_{4}\right)$ intoxication but before the final dose of $\mathrm{CCl}_{4}$, and another in which macrophages were depleted by diphtheria toxin administration only 3 days after the last dose of toxin was administered, at which time recovery from $\mathrm{CCl}_{4}$-mediated fibrosis had begun. These modest differences between the progression and recovery models of hepatic fibrosis in the timing of macrophage depletion (approximately 10 days) - specifically, the occurrence of depletion either before or after the final dose of $\mathrm{CCl}_{4}$ - yielded completely opposite effects on hepatic matrix accumulation. Depletion of macrophages while injury was progressing had an antifibrotic effect whereas depletion during early recovery led to sustained accumulation of matrix.

\section{Divergent phenotypes \\ of hepatic macrophages - one cell type or two?}

These results are fascinating, and they raise some important but unanswered questions regarding the underlying mechanisms. Are the same macrophages responsible for promoting matrix accumulation during progression and preventing it during recovery through phenotype switching, or are different subsets of macrophages recruited during this brief interval? While the former seems more likely and is consistent with evolving concepts $(7,15)$, an alternative explanation also supported by this (4) and much earlier studies (16) is the recruitment and differentiation of bone marrow-derived monocytes into hepatic macrophages. In fact, it is uncertain whether all these recruited monocytes become classic Kupffer cells or remain phenotypically distinguishable as a novel subpopulation. Regardless, if phenotypic conversion of the same cells accounts for this divergent behavior in progression versus recovery, what are the signals driving this switch? One approach to clarifying these questions would be to isolate CD11b-DTR-expressing cells by flow cytometry at different intervals during progression and recovery (without diptheria toxin administration) to analyze their transcriptional profile by microarray and to perform functional studies. This approach could be used in mice chimeric for bone marrow cells so that the phenotype of CD11b-DTR-expressing cells from liver could be distinguished from those derived from bone marrow.

How do the divergent macrophage phenotypes present during fibrosis progression versus recovery affect matrix production and degradation? In particular, are the effects of macrophage depletion mediated largely through their impact on activated stellate cells and/or myofibroblasts? During fibrosis progression, it is believed that macrophages are likely to promote activation of stellate cells through release of paracrine factors, including TGF- $\beta 1$, since this phenomenon has been demonstrated in culture $(17,18)$ (Figure 2$)$. In contrast, the simultaneous loss of macrophages (termed scar-associated macrophages by Duffield et al.; ref. 4) and activated stellate cells during recovery suggests that macrophages could also provoke apoptosis of hepatic stellate cells by the expression of TNF-related apoptosis-inducing ligand (known as TRAIL) and other apoptotic stimuli, as recently reported (19). Thus, the biphasic nature of macrophages could yield divergent effects on stellate cells, promoting their activation during progression and apoptosis during recovery. One potential regulator of this duality could be the cytokine TNF- $\alpha$ or its downstream signaling mediator NF- $\kappa \mathrm{B}$, since delicate temporal regulation of NF- $\mathrm{KB}$ signaling in hepatocytes can elicit profound and divergent effects on apoptosis and growth (20).

\section{Do hepatic macrophages degrade ECM during fibrosis regression?}

A more compelling question is whether macrophages directly promote matrix degradation during resolution of liver fibrosis through production of matrix-degrading proteases or by stimulating either the release or activation of proteases by other cell types, including stellate cells. Identifying macrophages as the elusive source of enzymes that degrade the fibrillar interstitial (i.e., scar) matrix during fibrosis resolution would represent a major advance, although the primary substrate for the hepatic macrophage's major known metalloproteinase, MMP-9, is basement-membrane collagen, not fibrillar collagen (21). Still, more careful characterization of recovery-associated macrophages could yield some surprises, including macrophage release of additional proteases or the existence of a more broad substrate specificity of MMP-9 that also includes fibrillar collagens; moreover, recent data suggest that activated stellate cells may also express MMP-9 (22). Finally, degradation of fibrillar ECM by recoveryassociated macrophages may yield an altered extracellular milieu that no longer supports survival of activated stellate cells, since breakdown of interstitial collagen represents loss of an important survival signal (23).

In summary, the exciting study by Duffield and colleagues (4) offers a new perspective in the study of hepatic injury and repair by resurrecting the prominence of hepatic macrophages, illustrating their dual phenotype in fibrosis progression and recovery, and highlighting their importance in regulating hepatic matrix degradation.

Address correspondence to: Scott L. Friedman, Box 1123, Mount Sinai School of Medicine, 1425 Madison Avenue, Room 1170C, New York, New York 10029, USA. Phone: (212) 659-9501; Fax: (212) 8492574; E-mail: Scott.Friedman@mssm.edu.

1. Wisse, E., Knook, D.L., and McCuskey, R.S. 1990. Fifth international symposium on cells of the bepatic sinusoid. The Kupffer Cell Foundation. Tuscon, Arizona, USA. 558 pp.

2. Pinzani, M., and Rombouts, K. 2004. Liver fibrosis: from the bench to clinical targets. Dig. Liver Dis. 36:231-242.

3. Friedman, S.L. 2004. Mechanisms of hepatic fibrosis and therapeutic implications. Nature Clinical Practice in Gastroenterology « Hepatology. 1:98-105.

4. Duffield, J.S., et al. 2005. Selective depletion of macrophages reveals distinct, opposing roles during liver injury and repair. J. Clin. Invest. 115:56-65. doi:10.1172/JCI200523928.

5. Decker, K. 1990. Biologically active products of stimulated liver macrophages (Kupffer cells). Eur. J. Biochem. 192:245-261. 
6. Wake, K., et al. 1989. Cell biology and kinetics of Kupffer cells in the liver. Int. Rev. Cytol. 118:173-229.

7. Goerdt, S., and Orfanos, C.E. 1999. Other functions, other genes: alternative activation of antigenpresenting cells. Immunity. 10:137-142.

8. Knolle, P.A., and Gerken, G. 2000. Local control of the immune response in the liver. Immunol. Rev. 174:21-34.

9. Vazquez-Torres, A., et al. 2004. Toll-like receptor 4 dependence of innate and adaptive immunity to Salmonella: importance of the Kupffer cell network. J. Immunol. 172:6202-6208.

10. Sun, Z., et al. 2003. Hepatic allograft-derived Kupffer cells regulate $\mathrm{T}$ cell response in rats. Liver. Transpl. 9:489-497.

11. Iredale, J.P., et al. 1998. Mechanisms of spontaneous resolution of rat liver fibrosis. Hepatic stellate cell apoptosis and reduced hepatic expression of metalloproteinase inhibitors. J. Clin. Invest. 102:538-549.

12. Issa, R., et al. 2004. Spontaneous recovery from micronodular cirrhosis: evidence for incomplete resolution associated with matrix cross-linking. Gastroenterology. 126:1795-1808.

13. Murphy, F.R., et al. 2002. Inhibition of apoptosis of activated hepatic stellate cells by tissue inhibitor of metalloproteinase- 1 is mediated via effects on matrix metalloproteinase inhibition: implications for reversibility of liver fibrosis. J. Biol. Chem. 277:11069-11076

14. Parsons, C.J., et al. 2004. Antifibrotic effects of a tissue inhibitor of metalloproteinase- 1 antibody on established liver fibrosis in rats. Hepatology. 40:1106-1115.

15. Duffield, J.S. 2003. The inflammatory macrophage: a story of Jekyll and Hyde. Clin. Sci. 104:27-38.

16. Gale, R.P., Sparkes, R.S., and Golde, D.W. 1978 Bone marrow origin of hepatic macrophages (Kupffer cells) in humans. Science. 201:937-938.

17. Matsuoka, M. and Tsukamoto, H. 1990. Stimulation of hepatic lipocyte collagen production by Kupffer cell-derived transforming growth factor beta: implication for a pathogenetic role in alcoholic liver fibrogenesis. Hepatology. 11:599-605

18. Friedman, S.L., and Arthur, M.J. 1989. Activation of cultured rat hepatic lipocytes by Kupffer cell conditioned medium. Direct enhancement of matrix synthesis and stimulation of cell proliferation via induction of platelet-derived growth factor receptors. J. Clin. Invest. 84:1780-1785.
19. Fischer, R., Cariers, A., Reinehr, R., and Haussinger, D. 2002. Caspase 9-dependent killing of hepatic stellate cells by activated Kupffer cells. Gastroenterology. 123:845-861.

20. Pikarsky, E., et al. 2004. NF-kappaB functions as a tumour promoter in inflammation-associated cancer. Nature. 431:461-466.

21. Knittel, T., et al. 1999. Expression patterns of matrix metalloproteinases and their inhibitors in parenchymal and non-parenchymal cells of rat liver: regulation by TNF-alpha and TGF-beta1. J. Hepatol. 30:48-60.

22. Han, Y.P., et al. 2004. Essential role of matrix metalloproteinases in interleukin-1-induced myofibroblastic activation of hepatic stellate cell in collagen. J. Biol. Chem. 279:4820-4828.

23. Issa, R., et al. 2003. Mutation in collagen-1 that confers resistance to the action of collagenase results in failure of recovery from $\mathrm{CCl} 4$-induced liver fibrosis, persistence of activated hepatic stellate cells, and diminished hepatocyte regeneration. FASEB J. 17:47-49.

24. Friedman, S.L. 2000. Molecular regulation of hepatic fibrosis, an integrated cellular response to tissue injury. J. Biol. Chem. 275:2247-2250.

\title{
Stay tuned to PXR: an orphan actor that may not be D-structive only to bone
}

\author{
Michael F. Holick
}

Vitamin D, Skin, and Bone Research Laboratory, Section of Endocrinology, Diabetes, and Nutrition, Department of Medicine, Boston University School of Medicine, Boston, Massachusetts, USA.

\begin{abstract}
Pregnane $X$ receptor (PXR) plays an important role in detoxifying xenobiotics and drugs. In this issue of the JCI, Pascussi et al. (see the related article beginning on page 177) provide convincing evidence that PXR can also induce vitamin $\mathrm{D}$ deficiency and bone disease because of its ability to cross-talk with the vitamin D-responsive gene that catabolizes 25-hydroxyvitamin $\mathrm{D}$ and 1,25-dihydroxyvitamin $\mathrm{D}$. This cross-talk behavior has important health ramifications and can be mitigated through the identification and treatment of PXR-induced vitamin D deficiency.
\end{abstract}

\section{Historical perspective}

In 1967 Schmid (1) reported an association between osteomalacia and antiepileptic drug therapy. This was confirmed by Dent et al. (2), who noted that osteomalacia and rickets were common in patients on longterm antiepileptic drug therapy, especially those who had been institutionalized and treated with multiple drugs to control their seizure disorder. Since these initial observations were made, there have been a multitude of reports of abnormalities in calcium,

Nonstandard abbreviations used: CYP24, 25-hydroxyvitamin D-24-hydroxylase; PXR, pregnane X receptor; $R X R$, retinoic acid $X$ receptor; VDR, vitamin $D$ receptor; VDRE, vitamin D-responsive element.

Conflict of interest: The author has declared that no conflict of interest exists.

Citation for this article: J. Clin. Invest. 115:32-34 (2005). doi:10.1172/JCI200523995. vitamin $\mathrm{D}$, and bone metabolism in subjects chronically treated not only with antiepileptic drugs but also with glucocorticoids, rifampin, and antiretroviral drugs (3-6). The disturbances observed in antiepileptic drug-treated patients were noted to be very similar to those of patients with vitamin D deficiency. More than $50 \%$ of children and adults receiving chronic antiepileptic drug therapy are at risk for developing abnormalities in calcium, vitamin $\mathrm{D}$, and bone metabolism (3). However, cardiac patients who had been treated with conventional doses of phenytoin for control of arrhythmias were found to be free of these abnormalities (7).

Antiepileptic drug-induced alterations in calcium and bone metabolism can include biochemical abnormalities such as hypocalcemia, hypophosphatemia, and elevated serum concentrations of alkaline phosphatase, parathyroid hormone, and 1,25-dihydroxyvitamin $\mathrm{D}\left[1,25(\mathrm{OH})_{2} \mathrm{D}\right]$. The biochemical hallmark for this disorder is reduced serum concentration of 25-hydroxyvitamin D [25(OH)D], the major circulating form, which is a barometer for a person's vitamin D status (8). There is an associated decrease in intestinal calcium absorption, a reduction in urinary calcium excretion, and an increase in bone turnover, as evidenced by increases in osteocalcin, bone-specific alkaline phosphatase, procollagen type $1 \mathrm{C}$-terminal extension peptide, and bone resorption markers including human collagen type $1 \mathrm{C}$-terminal peptide and total free deoxypyridinoline. The bone condition most often associated with chronic antiepileptic drug treatment is reduced bone mineral density and cortical bone loss. Rickets and, according to histologic evidence, osteomalacia indicating a defect in bone mineralization are also accompanying problems (3).

\section{Mechanism for drug-induced disorders of bone metabolism}

A variety of mechanisms have been proposed for the antiepileptic drug-induced disorders in calcium and bone metabolism. Gascon-Barré et al. (3-5) and Hahn et 